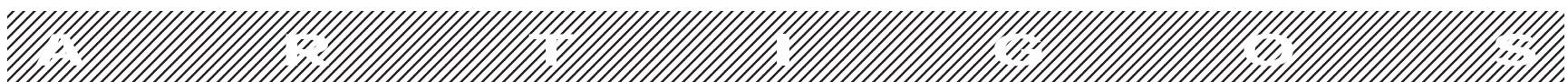

\title{
A Teoria das Facetas: noções básicas
}

\author{
Wolfgang Bilsky \\ Westfälische Wilhelms-Universität Münster
}

\begin{abstract}
Resumo
A Teoria das Facetas é um procedimento de pesquisa metateórico. Ela oferece um marco de referência formal que facilita o desenvolvimento de teorias e o estabelecimento de hipóteses, utiliza métodos que requerem um mínimo de restrições estatísticas e inter-relaciona sistematicamente delineamento de pesquisa, coleta de dados e análise estatística. Neste artigo esboçam-se os componentes básicos desta teoria, ilustra-se a aplicação da análise da estrutura de similaridades - um método freqüentemente empregado no contexto da Teoria das Facetas - e esquematizam-se os diversos papéis que as facetas desempenham na análise multidimensional. Isto é realizado usando como ilustração exemplos advindos da pesquisa de motivação e de valores, bem como estudos sobre medo do delito.
\end{abstract}

Palavras-chave: teoria das facetas; delineamento da pesquisa; escalonamento multidimensional; hipóteses regionais

\begin{abstract}
Facet Theory: basic notions. Facet Theory is a meta-theoretical approach to research. It offers a formal frame of reference for theory construction and hypothesis building, uses methods that require a minimum of statistical assumptions, and interrelates research design, data collection and statistical analysis systematically. In this article, the basic components of Facet Theory are outlined, the application of Similarity Structure Analysis, a method frequently used in the context of Facet Theory, is illustrated, and the different roles that facets are likely to play in multidimensional analysis are sketched out. This is accomplished by using examples from motivational and values research, as well as studies on fear of crime.
\end{abstract}

Key words: Facet Theory, research design, multidimensional scaling, regional hypotheses

A Teoria das Facetas (TF) recorre à teoria dos conjuntos. Segundo Coombs (1983), a primeira foi, no passado, o único esforço significativo de ocuparse de maneira crítica da tarefa complexa de identificar os limites de um setor específico do comportamento (ver Dancer, 1990, p. 367). Como suposição fundamental, a TF parte do pressuposto de que nas pesquisas empíricas na maioria das vezes não interessam quaisquer variáveis concretas, mas sim o universo das variáveis que representam. Ou seja, quando se define o campo de interesse de modo universal, tanto os sujeitos participantes nas pesquisas quanto as operacionalizações levadas a cabo (estímulos, itens, perguntas, tarefas) figuram somente como amostras do universo correspondente. Ademais, esta suposição implica que - independentemente dos instrumentos empregados pode-se comparar imediatamente os resultados de pesquisa, contanto que estes instrumentos apliquem as mesmas facetas, isto é, as mesmas categorias de conteúdo, para a classificação das observações empíricas.

A TF é um procedimento de pesquisa que abarca três aspectos diferentes. Primeiro, oferece princípios sobre como delinear pesquisas para a coleta sistemática dos dados. Igualmente, oferece um marco de referência formal que facilita o desenvolvimento de teorias. Neste sentido, é um procedimento metateórico. Segundo, apresenta uma variedade de métodos para analisar dados, métodos esses que se destacam por um mínimo de restrições estatísticas. Por este motivo, apresentam-se como adequados para analisar uma grande variedade de variáveis psicológicas e sociais. Finalmente, permite relacionar sistematicamente 0 delineamento da pesquisa, o registro dos dados e a sua análise estatística. Dito de outra forma, facilita expressar suposições teóricas, isto é, hipóteses, de tal forma que se pode examinar empiricamente a sua validade. 
Na realidade, a Teoria das Facetas não é um enfoque novo, visto que foi criada e desenvolvida por Louis Guttman durante os anos cinqüenta do século passado, entretanto são poucos os livros básicos ou publicações especializadas (por exemplo, Borg \& Shye, 1995; Canter, 1985; Dancer \& Hans, 1990; Guttman \& Greenbaum, 1998; Shye \& Elizur, 1994). O fato de o grupo dos pesquisadores que aplicou os princípios da TF em suas pesquisas psicológicas no passado ser relativamente pequeno se deve possivelmente a uma terminologia que, pelo menos à primeira vista, parece um pouco estranha. No Brasil, também se observa reduzido número de pesquisadores, sendo possível citar as publicações de Roazzi e colaboradores (Roazzi, 1995; Roazzi \& Monteiro, 1995; Roazzi \& Souza, 1997). Ademais, os programas necessários para realizar as análises estatísticas conforme a TF eram menos acessíveis em comparação com aqueles requeridos por outros enfoques metodológicos. Por fim, a difusão de uma idéia não depende somente da sua utilidade, mas também de modismos e casualidades.

Atualmente, o acesso ao software tornou-se pouco problemático; por exemplo, tanto o SPSS quanto o SYSTAT oferecem programas adequados. Além disso, a Teoria das Facetas demonstrou ser eficaz na planificação, realização e avaliação de um grande número de pesquisas empíricas nas ciências sociais (ver, por exemplo, Guttman \& Greenbaum, 1998). Portanto, uma maior difusão desta abordagem parece útil.

Nas páginas seguintes, esboçam-se algumas informações básicas a respeito da Teoria das Facetas (TF), relacionandoas com os três aspectos acima mencionados. Primeiro, explicase como especificar a posição de um problema de pesquisa e das hipóteses estruturais com a ajuda de facetas. Para responder à pergunta seguinte, qualquer que seja, se os dados empíricos refletem de fato as estruturas supostas previamente, necessita-se de métodos adequados. Entre aqueles desenvolvidos no contexto da TF, a Análise da Estrutura de Similaridades (Similarity Structure Analysis, SSA), uma forma especial de análises de Escalonamentos Multidimensionais (EMD), tornou-se muito útil em uma grande variedade de análises estruturais (Borg \& Shye, 1995; Canter, 1985; Shye \& Elizur, 1994). Portanto, na segunda parte analisam-se os elementos principais da sua aplicação. Na terceira parte, por fim, explica-se o papel das facetas neste tipo de análise. O emprego das facetas, a análise de escalonamento multidimensional e as suas inter-relações são ilustrados por intermédio de exemplos de pesquisa sobre a motivação, os valores e o medo do delito.

\section{Observações, facetas, elementos e sentença estruturadora}

Evidentemente, a faceta forma o núcleo deste enfoque metateórico. Essencialmente, corresponde à classificação de objetos e observações empíricas (variáveis) em categorias exclusivas e abrangentes com relação a um aspecto temático distinto estudado pelo pesquisador. Segundo esta especificação, uma faceta deve cobrir o aspecto temático em questão por completo e inequivocamente. As categorias que servem para classificar as observações são os elementos da faceta. Dito de outra forma, as facetas representam componentes conceituais não coincidentes do universo de interesse, de modo que se pode caracterizar cada variável por um (e exclusivamente um) elemento de cada faceta. De modo correspondente, quando se caracterizam os componentes do universo de interesse por $n$ facetas, cada variável deste universo é descrita por uma combinação de elementos das facetas consideradas, designada como n-tuple (teoria dos conjuntos) ou structuple (teoria das facetas; ver Dancer, 1990, p. 369).

Em resumo, diferenciam-se três tipos de facetas: o primeiro se refere à população dos sujeitos considerados na respectiva pesquisa; pode-se simbolizar este tipo com a letra $P$. O segundo concerne ao conteúdo das variáveis pesquisadas (estímulos, itens, perguntas), representado pela letra $C$. Juntas, as facetas de população e de conteúdo determinam o campo de interesse (domain of concern). Por fim, o terceiro tipo se refere ao universo das reações ou respostas dos sujeitos pesquisados. Para ser mais exato, refere-se ao conjunto das categorias exclusivas, disponíveis para avaliar as tarefas ou, simplesmente, os itens usados na pesquisa. Este tipo é nomeado como amplitude de respostas admissíveis, normalmente apresentadas como uma escala ordenada de aceitação e simbolizado pela letra $R$.

Fazendo observações empíricas, os conjuntos $P, C$ e $R$ estão associados sistematicamente entre si. Em termos da Teoria das Facetas, as observações se mostram como representações (mapping) do campo do interesse $P \times C$ na variedade $R$. Este tipo de sentença estruturadora se simboliza como $P \times C=>R$, em que o conjunto $P \times C$ corresponde ao produto cartesiano dos conjuntos $P$ e $C$. Formalizado desta maneira, cada observação corresponde à classificação de um elemento de $R$ por um elemento de $P$ x C. Por exemplo, numa situação específica de pesquisa (um tratamento experimental), cada participante (sujeito do experimento) é associado a um comportamento específico e admissível (reação), segundo o delineamento da pesquisa.

Para expressar um assunto de pesquisa nos termos da TF, normalmente são necessárias várias facetas, tanto de $P$ como de $C$ (às vezes se necessita também de mais de uma faceta de $R$ ).

Em pesquisas sociológicas, por exemplo, encontram-se muitas facetas do tipo $P$. Assim, além de uma faceta para o registro do sexo, há outras como o estado civil, a idade, a renda, a afiliação a um partido, etc. Enquanto os elementos das facetas sexo (masculino, feminino), estado civil (solteiro, casado, divorciado) e afiliação a um partido (A, B, ..., X) formam categorias nominais ou qualitativas, os elementos de idade e renda pertencem a facetas ordenadas, compreendendo uma amplitude de mínimo a máximo.

Em pesquisas psicológicas, por outro lado, interessam mais as facetas do tipo $C$. Assim, numa pesquisa experimental, por exemplo, poder-se-ia analisar o efeito comum de álcool (C1), peso corporal (C2) e cansaço (C3) sobre o tempo de reação $(R)$, usando um delineamento fatorial. Do mesmo modo, poder-se-iam efetuar entrevistas ou empregar questionários para conhecer a importância de variáveis como penas anteriores (C1), gravidade do dano (C2) e idade do delinqüente (C3) para a sanção penal adequada, de acordo com a opinião pública $(R)$. 


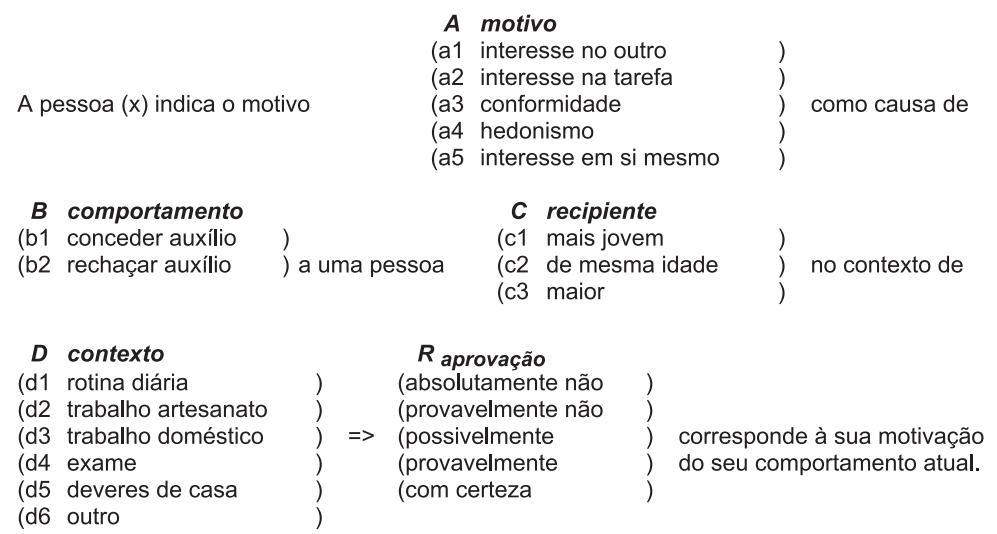

Figura 1. Sentença estruturadora - Questionário de Motivação Pró-Social (PSMQ; ver Bilsky, 1993)

Todo delineamento de facetas (facet design) define uma pesquisa específica - seja descritiva ou experimental - e pode resumir-se em uma sentença estruturadora (mapping sentence). Tal sentença não somente cita as diferentes facetas de interesse científico de modo aditivo, mas também especifica as suas relações mútuas, usando nexos como na língua falada. Em conformidade, lêem-se as sentenças estruturadoras “da esquerda para a direita”, aplicando a cada vez um elemento de cada faceta. Dessa maneira, resultam tantas frases quantas diferentes combinações existam entre os elementos pertencentes ao campo do interesse. Portanto, o universo de perguntas que requerem explicações numa pesquisa concreta é determinado inequivocamente pelas facetas e as suas interrelações, como especificadas pela sentença estruturadora.

Com o objetivo de ilustrar, recorremos a uma re-análise do Questionário de Motivação Pró-Social (Prosocial Motivation Questionnaire, PSMQ), que é fundamentado na teoria de Reykowski (Silbereisen, Boehnke \& Reykowski, 1986). Esta re-análise foi realizada com o objetivo de validar a estrutura deste instrumento (ver Bilsky, 1993). O princípio da construção do questionário pode ser resumido numa sentença estruturadora, como demonstrado na Figura 1. Observa-se que nesta sentença há quatro facetas do tipo $C$ definindo o conteúdo pesquisado: o motivo (A), o comportamento (B), o recipiente (C) e o contexto (D).
Partindo desta sentença, os itens do PSMQ podem ser analisados sistematicamente. A Tabela 1 exibe um item deste questionário, indicando a operacionalização das facetas baseadas na sentença estruturadora. Evidentemente, quando se emprega este instrumento em um estudo concreto, é indispensável que se especifique também a população dos participantes pesquisados, isto é, as facetas do tipo $P$.

Utilizando desta maneira as facetas e sua organização em frases estruturadoras, facilita-se de um lado a construção dos itens e de outro o registro das observações e a coleta dos dados de modo sistemático. Ademais, possibilita-se a revisão de instrumentos já existentes no que diz respeito à correspondência inequívoca entre a teoria original e a sua operacionalização, obrigando o revisor a identificar explicitamente os diferentes aspectos teóricos (i.e., as facetas) no instrumento (a utilidade deste procedimento também foi demonstrada na nossa re-análise; ver Bilsky, 1993).

Planejar sistematicamente a realização de um estudo é uma tarefa importante nas ciências empíricas. Sem dúvida, isto é apenas a primeira parte de uma investigação. No final, o que interessa é a constatação de que os dados colecionados refletem a teoria do pesquisador, ou seja, estruturam-se em conformidade com as categorias e suas inter-relações teoricamente deduzidas. Observa-se isso nos tipos de análise estrutural ilustrados nos parágrafos seguintes.

Tabela 1

Item do Questionário de Motivação Pró-Social (PSMQ; ver Bilsky, 1993)

Faz um bom tempo. Depois da escola você vai ver um amigo. Ele está ajudando seus pais na limpeza da casa [contexto]. Como ele vai demorar bastante para acabar com este trabalho, você decide ajudar [comportamento] seu amigo [recipiente].

Quais foram os seus motivos [norma] para tomar esta decisão?

(1) Depois de estar sentado durante todo o tempo na escola, acreditei que seria interessante trabalhar um pouco para sentir-me melhor [hedonismo].

(2) Dei-me conta de que teria de limpar o porão e pensei que o meu amigo também poderia ajudar-me com este trabalho [interesse em si mesmo].

(3) Sabia que, se eu o ajudasse, o trabalho terminaria mais rápido [interesse na tarefa].

(4) É claro que os amigos se ajudam mutuamente; caso contrário, não seriam verdadeiros amigos [interesse no outro]

(5) Como todo mundo estava ajudando, não quis ficar sentado sem fazer nada [conformidade]. 


\section{Análise da estrutura de similaridades}

Nas ciências sociais, empregam-se vários métodos para identificar e analisar as estruturas das relações entre as variáveis. Entre tais métodos encontram-se, por exemplo, a análise fatorial e a análise de cluster. A análise da estrutura de similaridades (SSA), embora menos conhecida, serve ao mesmo objetivo. Este método é também conhecido como análise do menor espaço (Smallest Space Analysis, ver Borg \& Groenen, 1997a).

Na SSA - que é uma forma não-métrica das análises de escalonamentos multidimensionais (EMD) - as (des)semelhanças entre as variáveis ou objetos se refletem como proximidades e distâncias entre pontos, de tal modo que variáveis semelhantes são agrupadas, enquanto as diferentes encontram-se distantes no espaço multidimensional. Isto se realiza de forma que os dados de (des-)semelhanças sejam funções monotônicas (ordenadas) das distâncias neste espaço. Em comparação com a análise fatorial, a SSA é menos restritiva a respeito das variáveis analisadas. Assim, não é necessário que os dados apresentem características métricas, tampouco os coeficientes de associação analisados pela SSA necessitam ser lineares; entretanto, tanto os coeficientes de associação lineares como os monotônicos são adequados para este tipo de análise.

Depois de fixar a dimensionalidade do espaço que serve para retratar a configuração das variáveis de interesse, a SSA determina as coordenadas para localizar estas variáveis como pontos no espaço multidimensional. Estes pontos marcam a localização de uma variável em relação ao total das outras variáveis pesquisadas. Diferente da análise fatorial, não se interpretam as dimensões que definem o espaço multidimensional; na verdade, as dimensões funcionam somente como meio para possibilitar a verificação de diferentes projeções da configuração total.
Por razões de economia, busca-se o menor espaço, isto é, o número mínimo de dimensões para representar as interdependências e associações mútuas. Para decidir sobre a conveniência da solução eleita, o pesquisador pode referirse - além da sua teoria - a dois critérios: o coeficiente de alienação e o diagrama de Shepard.

Ambos os critérios formam parte de uma variedade de indicadores de precisão de ajuste.

Outros indicadores empregados em EMD são o s-stress de Young, o estresse de Kruskal e a correlação múltipla ao quadrado. O coeficiente de alienação $K$ é um valor estatístico utilizado exclusivamente em EMD não-métrico, isto é, na SSA ordinal (Borg \& Shye, 1995). Normalmente, um coeficiente $K$ $\leq 0,15$ indica uma solução aceitável para interpretar os dados. De fato, esta é apenas uma regra geral, pois a conveniência de uma solução multidimensional depende de muitas considerações. Por exemplo, uma vez pressuposto que se analisa um grande número de variáveis em relação à dimensionalidade elegida numa SSA, pode-se tolerar um coeficiente de alienação mais elevado. Isto porque, no caso de muitos coeficientes representados no espaço multidimensional, a estrutura resulta mais estável e menos suscetível a variações aleatórias e erradas (ver Borg \& Groenen, 1997a). Afinal, são as considerações teóricas que decidem sobre a utilidade e adequação de uma solução multidimensional.

O diagrama de Shepard é outro meio para julgar a precisão de ajuste e detectar possíveis anomalias na representação multidimensional. Neste diagrama, os coeficientes de (des)semelhança e as distâncias respectivas são reproduzidas em pares. Para que a configuração esteja bem ajustada, este diagrama deve refletir uma função crescente ou decrescente sem interrupções ou descontinuidades. Dependendo do tipo de indicador de (des-)semelhança empregado (e.g., coeficiente de correlação) encontra-se, por exemplo, uma configuração

Tabela 2

Correlações entre dez tipos distintos de valores de acordo com a teoria de Schwartz (1992)

\begin{tabular}{|c|c|c|c|c|c|c|c|c|c|c|}
\hline & $\mathrm{PO}$ & EX & $\mathrm{HE}$ & $\mathrm{ES}$ & $\mathrm{AD}$ & UN & $\mathrm{BE}$ & TR & $\mathrm{CO}$ & SE \\
\hline Poder & - & . & . & . & . & . & . & . & . & . \\
\hline Êxito & 0,58 & - & . & . & . & . & . & . & . & . \\
\hline Hedonismo & 0,21 & 0,13 & - & . & . & . & . & . & . & . \\
\hline Estimulação & 0,15 & 0,23 & 0,35 & - & . & . & . & . & . & . \\
\hline Autodireção & $-0,02$ & 0,25 & 0,18 & 0,38 & - & . & . & . & . & . \\
\hline Universalismo & $-0,40$ & $-0,22$ & 0,02 & 0,15 & 0,23 & - & . & . & . & . \\
\hline Benevolência & $-0,14$ & $-0,02$ & 0,14 & 0,09 & 0,01 & 0,42 & - & . & . & . \\
\hline Tradição & 0,03 & $-0,06$ & $-0,16$ & $-0,06$ & $-0,06$ & 0,25 & 0,18 & - & . & . \\
\hline Conformidade & 0,27 & 0,26 & 0,00 & $-0,05$ & $-0,07$ & 0,09 & 0,28 & 0,48 & - & . \\
\hline Segurança & 0,33 & 0,28 & 0,14 & 0,00 & $-0,06$ & 0,07 & 0,23 & 0,32 & 0,58 & - \\
\hline
\end{tabular}


crescente ou decrescente. Um ajuste perfeito, portanto, manifesta-se através de uma diagonal no diagrama de Shepard.

O exemplo seguinte ilustra a análise da estrutura de similaridades. Os dados são provenientes de um estudo sobre estruturas comuns de motivos e valores (Bilsky, 1999a). Trata-se da análise de correlações entre dez tipos distintos de valores (Tabela 2) para, nesse contexto, verificar a estrutura circular prognosticada pela teoria de Schwartz (1992) sobre os tipos de valores.

Uma SSA bidimensional desta matriz de correlações resultou em um coeficiente de alienação $K=0,12$; o diagrama de Shepard correspondente é reproduzido na Figura 2.

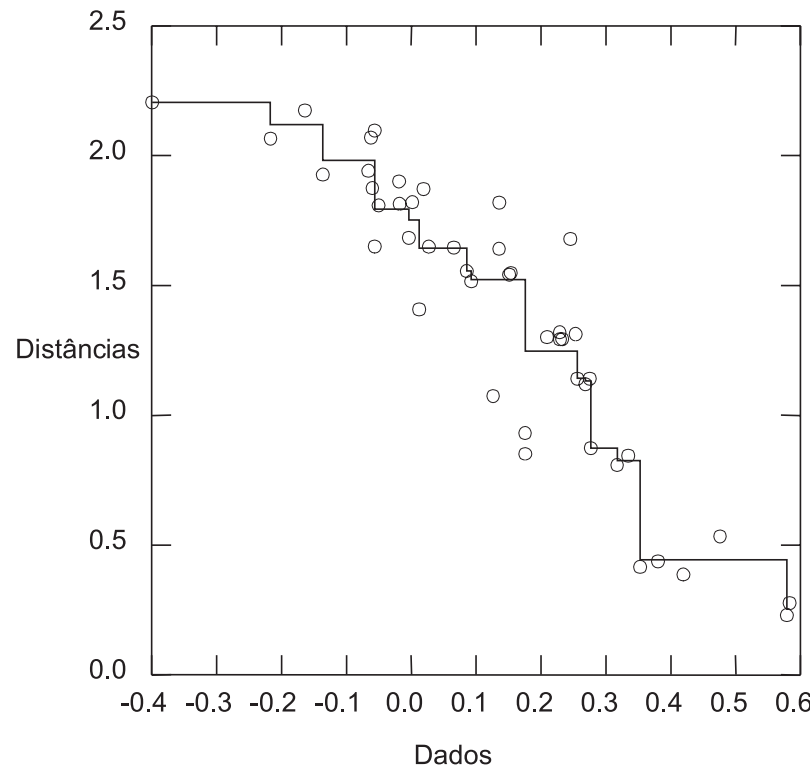

Figura 2. Diagrama de Shepard de uma SSA bidimensional de dez tipos de valores segundo a teoria de Schwartz (1992).

Tanto o coeficiente de alienação quanto o diagrama de Shepard indicam uma solução aceitável para representar a estrutura dos valores num espaço de duas dimensões. Como se pode verificar na Figura 3, a configuração circular encontrada nestes dados corresponde muito precisamente ao modelo de Schwartz (1992). De acordo com esta forma de circumplex, a maioria das correlações mais elevadas encontrase próxima da diagonal principal da matriz na Tabela 2, isto é, entre os valores vizinhos da Figura 3; os coeficientes baixam

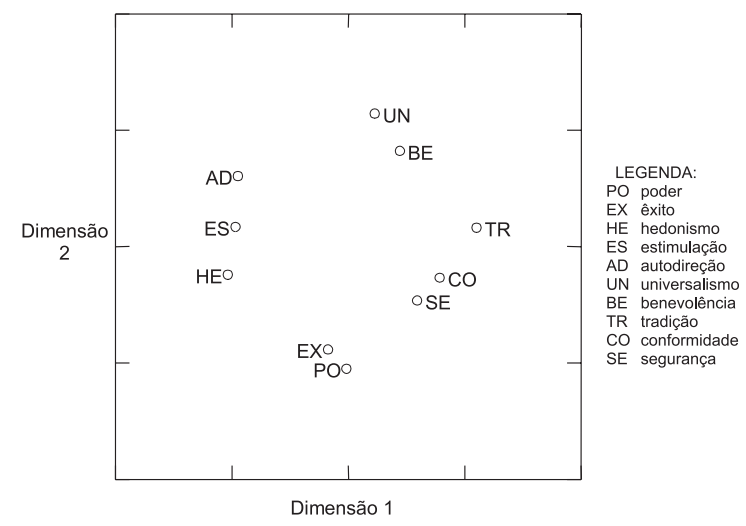

Figura 3. Configuração de dez tipos distintos de valores, segundo Schwartz (1992). depois e aumentam de novo quando se aproximam do ângulo inferior esquerdo da matriz. Não obstante, a SSA não se baseia somente em correlações isoladas, mas sim no conjunto de todos os coeficientes.

Até agora esboçaram-se alguns aspectos técnicos da SSA. O que falta, todavia, são informações sobre como relacionar as facetas e os dados de modo sistemático. Em outras palavras, faltam informações sobre o papel específico das facetas na estruturação do espaço multidimensional, o que será discutido na última parte deste texto.

\section{O papel das facetas em uma análise da estrutura de similaridades}

As facetas de conteúdo (domain facets) da frase estruturadora formam a base para formular hipóteses estruturais, isto é, hipóteses regionais com relação à solução esperada. De fato, a Teoria das Facetas parte da suposição de que as facetas têm um papel específico na estruturação do espaço multidimensional. Assim, a identificação de regiões no espaço multidimensional depende do tipo de correspondência entre a sentença estruturadora, que define o campo de interesse (isto é, o universo de observações) e o espaço SSA, que representa a inter-relação das variáveis como uma amostra do universo. Neste contexto, uma região é especificada para um determinado subconjunto de variáveis, identificando-as por um elemento comum pertencente a uma das facetas incluídas na sentença estruturadora. Estas regiões tomam formas muito específicas, como configurações circulares, cuneiformes ou faixas paralelas.

No passado, identificaram-se várias vezes as correspondências entre tipos distintos de facetas de conteúdo e configurações de variáveis no espaço multidimensional (Levy, 1985). Para ser exato, é muito freqüente que se encontre três protótipos de partição, conhecidos na literatura como axial, modular (ou radial) e polar (ou angular; ver Borg \& Shye, 1995; Dancer, 1990). Portanto, parece apropriado descrever estas formas brevemente (ver Figura 4, que oferece uma orientação geral sobre estes tipos de partição).

Para começar, a forma de separação encontrada numa análise multidimensional depende do fato de ela resultar de facetas ordenadas ou qualitativas (categoriais). Qualifica-se uma faceta como ordenada quando se pode agrupar seus elementos de forma a que cada elemento seguinte represente a respectiva característica de modo progressivo. Quando se trata de uma faceta ordenada é possível prognosticar a hierarquia de correlações entre pares de variáveis.

Uma faceta ordenada pode fazer um papel axial ou modular ao dividir o espaço multidimensional, dependendo de sua relação com as outras facetas na sentença estruturadora (Dancer, 1990). Se não tem relações com outras facetas, a faceta ordenada se apresentará de modo axial, ou seja, seus elementos se manifestarão em sucessão linear, separados por linhas paralelas. Este tipo de partição é também conhecido como simplex axial de regiões (Borg \& Shye, 1995). 
Por outro lado, quando a faceta ordenada encontra-se relacionada com uma ou mais facetas, seus elementos se manifestarão como círculos concêntricos, isto é, de forma modular. Nesse caso, as variáveis representadas por pontos no círculo central têm um sentido mais geral que aquelas que estão localizadas próximas à borda. Dito de outro modo, as variáveis mais periféricas têm algo em comum com as variáveis centrais, mas também possuem algo de específico (Dancer, 1990). Nesse sentido, as correlações das variáveis no centro resultarão mais altas, diminuindo à medida que se afastam.

Além das facetas ordenadas, existem outras cujos elementos se diferenciam de modo qualitativo, sem que manifestem (normalmente) qualquer ordem óbvia. Estas facetas têm um papel polar (angular), isto é, seus elementos constituem regiões cuneiformes, de forma circumplex, com limites partindo de uma origem comum (Borg \& Shye, 1995). Neste tipo de separação, os elementos de regiões adjacentes mostrarão uma semelhança maior com relação à característica medida para a faceta correspondente do que os de regiões não-adjacentes.
Papel das Facetas

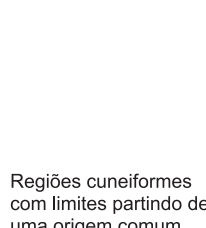

Polar uma origem comum

Partição do Espaço Círculos concêntricos comum

Faixas paralelas ao redor de um centro
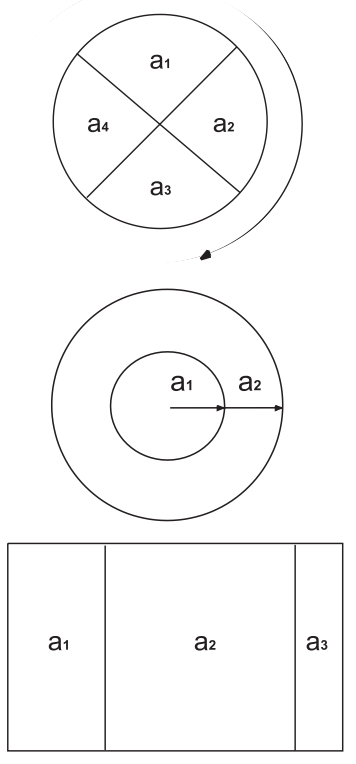

Figura 4. Papel das facetas e partição do espaço multidimensional, segundo Levy (1985).

Partindo desses papéis básicos das facetas, podem-se identificar várias separações mais complexas do espaço multidimensional. Por exemplo, um duplex resulta da combinação de duas facetas axiais, representadas num espaço bidimensional. Outra configuração bidimensional resulta da combinação de uma faceta modular (caracterizada por limites concêntricos) com outra de forma polar (isto é, com limites de origem comum); esta separação é designada radex. A configuração mais freqüentemente identificada como um espaço tridimensional é chamada cylindrex. Resulta da combinação de um radex com uma separação axial na terceira dimensão. A Figura 5 serve como exemplo para se visualizar uma configuração complexa bidimensional.

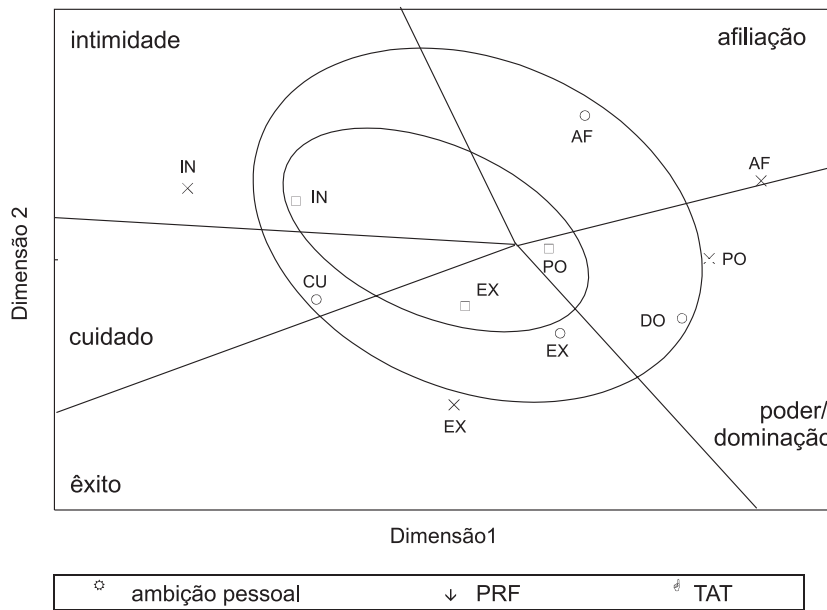

Figura 5. Radex: relação entre diferentes tipos de motivos (Emmons \& McAdams, 1991; ver Bilsky, 1999a).

Como se pode observar, os dados re-analisados aqui se mostram como um radex. Eles são provenientes de um estudo de Emmons e McAdams (1991) em que os autores pesquisaram a relação entre diferentes tipos de motivos afiliação, poder, dominação, êxito, cuidado e intimidade medidos por três instrumentos: Ambições Pessoais (personal strivings), PRF (Personality Research Form) e TAT (Teste de Apercepção Temática). O total das correlações desta pesquisa forma uma matriz do tipo multi-trait-multi-method (MTMM). Segundo Borg e Groenen (1997b), este tipo de matriz pode ser analisada efetivamente com a ajuda de EMD. Em uma matriz MTMM, os traços (traits) e os métodos correspondem a duas facetas que se utilizam para classificar os itens ou as variáveis. De acordo com esta interpretação, a re-análise dos dados de Emmons e McAdams com a ajuda da SSA resultou numa partição perfeita de conteúdo (características pessoais) e método (para mais detalhes, ver Bilsky, 1999a).

Apesar de se poder empregar a SSA, assim como a EMD em geral, como um dos vários métodos para analisar dados empíricos, o seu potencial com respeito à identificação de estruturas teóricas será mais óbvio se em combinação com um delineamento de facetas. Na verdade, a SSA torna possível a análise confirmatória para comprovar a validade de hipóteses regionais derivadas das facetas e suas relações, conforme especificadas na sentença estruturadora, isto é, no delineamento das facetas. Para mostrar a relação simétrica entre o delineamento das facetas e a análise da estrutura de similaridades, relata-se a seguir um estudo planejado e avaliado por meio da TF.

Este estudo compreendeu parte de um projeto mais complexo sobre "sentimento de segurança pessoal, medo do delito e da violência, e vitimização das pessoas idosas” (Bilsky, Pfeiffer, \& Wetzels, 1993; Bilsky, 1999b) e pesquisou a estrutura multidimensional dos fatores criminais e nãocriminais que afetam a segurança pessoal, segundo as percepções subjetivas na população alemã. A investigação partiu da evidência de que vários fatores individuais e sociais podem ameaçar os sentimentos de segurança pessoal - um 


\section{A pessoa $(x)$ sente-se em tensão por uma lesão potencial}

\author{
A lesão \\ (a1 material \\ (a2 física \\ (a3 psicológica \\ (a4 não especificada
}

B ambiente

(b1 primário

(b2 secundário

(b3 não especificado
)

) como conseqüência de acontecimentos no seu ) )

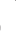

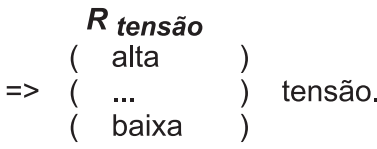

C espaço vital

(c1 saúde

(c2 trabalho

(c3 economia

(c4 social

(c5 residência

(c6 não especificado

Figura 6. Sentença de representação: tensão no indivíduo por causa da discrepância percebida entre o status desejado de segurança pessoal e a realidade.

termo que surge todos os dias na linguagem leiga. Esses fatores podem ser considerados como estressantes, uma vez que provocam tensão no indivíduo por causa da discrepância percebida entre o status desejado de segurança pessoal e a realidade. Visto que a ausência de tensão é um traço fundamental e definidor do bem-estar subjetivo, pesquisas sobre este tema serviram como base para especificar as facetas que provavelmente se mostram úteis e eficazes para distinguir sistematicamente os fatores estressantes da segurança pessoal. Neste contexto, as pesquisas de Levy e Guttman (1989) foram de interesse especial, pois os autores desenvolveram e validaram uma sentença estruturadora geral, integrando o estresse, o coping e o bem-estar. Orientando- nos por sua definição formal, nossas facetas se integraram dentro da sentença estruturadora reproduzida na Figura 6.

Com base nesta definição, foram considerados e classificados, num passo anterior às análises estatísticas, vários fatores estressantes de acordo com as facetas diferenciadas na sentença estruturadora. A Figura 7 mostra três fatores estressantes que servem como exemplos, classificados conforme as facetas lesão, ambiente e espaço vital.

Com referência ao papel das facetas na SSA, em geral, e às pesquisas de Levy e Guttman (1989) em particular, poderse-ia antecipar que as facetas lesão e espaço vital desempenhariam um papel polar (angular), e o ambiente um papel modular. As análises multidimensionais (SSA), tanto

Pergunta: Em que medida você sente que a sua segurança pessoal está ameaçada pelos seguintes acontecimentos e perigos?

item structuples

estressantes:

inflação e crise econômica

doença grave

enfados e disputas familiares

...

facetas e elementos das facetas: a1 b2 c3

a2 b1 c1

a3 b1 c4

$$
\begin{aligned}
& \text { B ambiente } \\
& \text { b1 }=\text { primário } \\
& \text { b2 }=\text { secundário } \\
& \text { b3 }=\text { não especificado }
\end{aligned}
$$$$
\text { a3 = psic ológica }
$$$$
\text { a4 = não especificada }
$$

$$
\begin{aligned}
& \boldsymbol{C} \quad \text { espaço vital } \\
& \text { c1 }=\text { saúde } \\
& \mathrm{c} 2=\text { trabalho } \\
& \mathrm{c} 3=\text { economia } \\
& \mathrm{c} 4=\text { social } \\
& \mathrm{c5}=\text { residência } \\
& \mathrm{c} 6=\text { não especificado }
\end{aligned}
$$

Figura 7. Fatores estressantes, classificados conforme as facetas lesão, ambiente e espaço vital. 
de um estudo piloto quanto de uma pesquisa representativa, confirmaram estas hipóteses regionais. Destacaram também a utilidade deste delineamento conceitual para pesquisar delitos criminais em relação a outros fatores estressantes, não delitivos, do bem-estar psicológico. Por exemplo, é possível mostrar que a delinqüência em geral se localiza no ambiente secundário, sugerindo uma interpretação sistêmica deste fator estressante, ou seja, uma interpretação que se pode chamar mais adequadamente de preocupação pela delinqüência (Skogan, 1993). Em contraste, os atos delitivos mais concretos analisados nesta pesquisa (assalto, roubo, fraude) surgiram no ambiente primário. Portanto, entende-se mais como medo pessoal do delito. Esta não é somente uma diferenciação terminológica, mais que isso, reflete-se muito diretamente nos dados de pesquisas criminológicas que dizem respeito aos sentimentos de segurança pessoal (Bilsky, Pfeiffer, \& Wetzels, 1993; Skogan, 1993). Como ilustração, a Figura 8 resume os resultados do estudo que explora a localização da delinqüência em geral (Bilsky, 1999b).

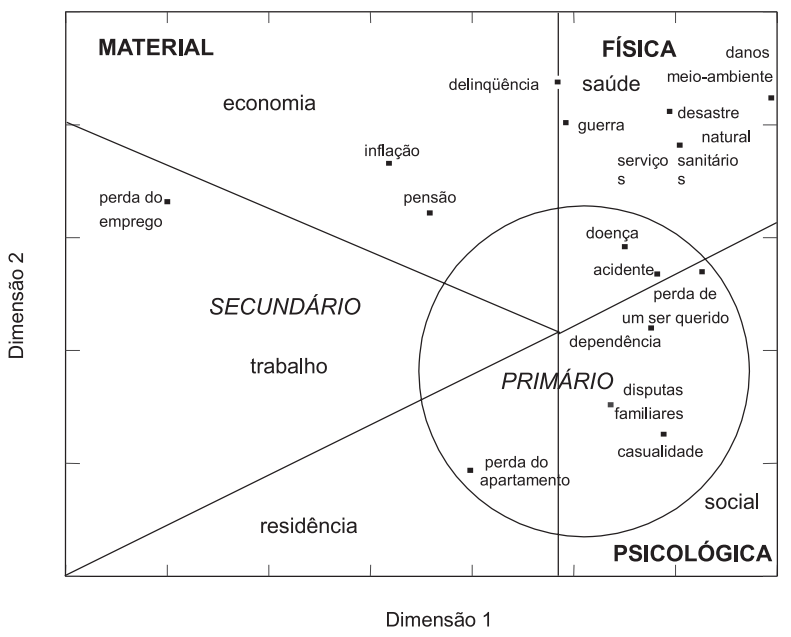

Figura 8. Configuração de fatores estressantes: SSA bidimensional.

Em resumo, o presente estudo demonstrou como aplicar a TF para: (1) definir o campo de interesse para a especificação das facetas teoricamente relevantes e as suas inter-relações numa frase estruturadora; (2) formular hipóteses regionais a fim de que se possa verificar a validade da facetização elegida; (3) realizar uma análise confirmatória, empregando a SSA como método.

\section{Conclusão}

Evidentemente, neste texto somente se podem apresentar aspectos muito básicos da TF. Não obstante, parece oportuno oferecer algumas conclusões gerais, e indicar perspectivas para a sua aplicação.

Pode-se, em primeiro lugar, constatar que a TF, como conseqüência de sua formalização extensa, obriga os pesquisadores, não só a apresentar as suas suposições teóricas claramente, como também a operacionalizar as variáveis dependentes e independentes de modo inequívoco, o que se torna evidente ao tentarem condensar suas hipóteses em sentenças estruturadoras. Estas definições formais têm uma posição central com vistas à planificação e avaliação das pesquisas empíricas. Marcam o domínio de validade de cada pesquisa e indicam como investigar as hipóteses de modo adequado, uma vez que, por causa dos papéis específicos das facetas nas análises multidimensionais, existem relações diretas entre as hipóteses regionais e os métodos desenvolvidos e empregados no contexto da TF. Em outras palavras, a TF é uma abordagem sistemática, integrando tanto o delineamento quanto a avaliação das pesquisas de modo simétrico. Assim, a TF se mostra como modelo metateórico para desenvolver ou melhorar os seus próprios modelos e teorias de pesquisa.

Em segundo lugar, pode-se empregar efetivamente a TF como meio didático nos cursos metodológicos. De fato, ela tem freqüentemente se mostrado uma ajuda muito eficaz na instrução de alunos iniciantes, porque acentua de modo transparente a necessidade de operacionalizar claramente suas pressuposições, tanto no que concerne às variáveis empregadas, quanto com respeito ao delineamento da pesquisa. Além disso, é possível compreender os princípios dos métodos empregados na TF, especialmente os princípios da SSA, sem conhecimento aprofundado dos métodos estatísticos. Dessa maneira, as relações entre os problemas científicos e os métodos de pesquisa podem ser demonstradas desde o princípio dos estudos.

Finalmente, existem outras formas de análises multidimensionais que complementam os métodos aqui esboçados e que abrem perspectivas adicionais ao pesquisador avançado. Assim, tanto a multidimensional structuple analysis (MSA) quanto a partial order structuple analysis (POSA) oferecem oportunidades suplementares para analisar dados (Borg \& Groenen, 1997a, Shye, 1985). Seguramente, não é por acaso que o SYSTAT integrou também o partially ordered scalogram analysis with coordinates (POSAC) em sua oferta de programas.

De qualquer modo, vale à pena avaliar criticamente as vantagens e desvantagens dos tipos de delineamento e de análise multidimensional aqui descritos em comparação com os métodos mais conhecidos.

\section{Agradecimentos}

O presente manuscrito originou-se de uma estada do autor como pesquisador visitante na Universidade Federal da Paraíba, João Pessoa, Brasil (CNPq, Processo 453699/01-4, NV). Sou muito grato aos professores Valdiney V. Gouveia e Antonio Roazzi, por ajudarem na revisão do texto.

\section{Referências}

Bilsky, W. (1993). Assessing prosocial motivation. The German Journal of Psychology, 17, 1-10.

Bilsky, W. (1999a). Common structures of motives and values: towards a taxonomic integration of two psychological constructs (Berichte aus dem 
Psychologischen Institut IV, $\mathrm{n}^{\underline{0}}$ 19). Münster, Alemanha: Westfälische Wilhelms-Universität.

Bilsky, W. (1999b). Miedo al delito, victimización criminal, y la relación miedo-victimización: algunos problemas conceptuales y metodológicos. In L. Arroyo, J. Montanés \& C. Rechea (Orgs.), Estudios de Criminología II (pp. 313-328). Castilla-La Mancha: Ediciones de la Universidad.

Bilsky, W., Pfeiffer, C., \& Wetzels, P. (1993). Feelings of personal safety, fear of crime and violence, and the experience of victimization amongst elderly people. In W. Bilsky, C. Pfeiffer \& P. Wetzels (Orgs.), Fear of crime and criminal victimization (pp. 245-267). Stuttgart: Enke.

Borg, I., \& Groenen, P. (1997a). Modern multidimensional scaling. Theory and applications. Berlim: Springer.

Borg, I., \& Groenen, P. (1997b). Multitrait-multimethod by multidimensional scaling. In W. Bandilla \& F. Faulbaum (Orgs.), SoftStat '97. Advances in Statistical Software 6 (pp. 59-65). Stuttgart: Lucius \& Lucius.

Borg, I., \& Shye, S. (1995). Facet Theory: form and content. Newbury Park, Califórnia: Sage.

Canter, D. (Org.). (1985). Facet Theory. Nova York: Springer.

Coombs, C. (1983). Psychology and mathematics. Ann Arbor: The University of Michigan Press.

Dancer, L. S. (1990). Introduction to Facet Theory and its applications. Applied Psychology: An International Review, 39, 365-377.

Dancer, L. S., \& Hans, S. L. (Orgs.). (1990). Applied Psychology: An International Review [Special Issue], 39(4).

Emmons, R. A., \& McAdams, D. P. (1991). Personal strivings and motive dispositions: exploring the links. Personality and Social Psychology Bulletin, 17, 648-654.

Guttman, R., \& Greenbaum, C. W. (1998). Facet Theory: its development and current status. European Psychologist, 3, 13-36.

Levy, S. (1985). Lawful roles of facets in social theories. In D. Canter (Org.), Facet Theory. Approaches to social research (pp. 59-96). Nova York: Springer.
Levy, S., \& Guttman, L. (1989). The conical structure of adjustive behavior. Social Indicators Research, 21, 455-479.

Roazzi, A. (1995). Categorização, formação de conceitos e processos de construção de mundo: procedimento de classificações múltiplas para o estudo de sistemas conceituais e sua forma de análise através de métodos de análise multidimensionais. Cadernos de Psicologia, 1, 1-27.

Roazzi, A., \& Monteiro, C. M. G. (1995). A representação social da mobilidade profissional em função de diferentes contextos urbanos e suas implicações para a evasão escolar. Arquivos Brasileiros de Psicologia, 47(3), 39-73.

Roazzi, A., \& Souza, B. C. (1997). Epistemology of cognitive development. In A. Fogel, M. C. D. P. Lyra \& J. Valsiner (Orgs.), Dynamics and indeterminism in developmental and social processes (pp. 217-243). Hillsdale, New Jersey: Lawrence Earlbaum.

Schwartz, S. H. (1992). Universals in the content and structure of values: theoretical advances and empirical tests in 20 countries. In M. Zanna (Org.), Advances in Experimental Social Psychology, (Vol. 25, pp. 1-65). Nova York: Academic Press.

Shye, S. (1985). Multiple Scaling. The theory and application of Partial Order Scalogram Analysis. Amsterdam: North-Holland.

Shye, S., \& Elizur, D. (1994). Introduction to Facet Theory. Content design and intrinsic data analysis in behavior research. Thousand Oaks, Califórnia: Sage.

Silbereisen, R. K., Boehnke, K., \& Reykowski, J. (1986). Prosocial motives from 12 to 18: a comparison of adolescents from Berlin (West) and Warsaw. In R. K. Silbereisen, K. Eyferth \& G. Rudinger (Orgs.), Development as action in context (pp. 137-164). Berlim: Springer.

Skogan, W. G. (1993). The various meanings of fear. In W. Bilsky, C. Pfeiffer \& P. Wetzels (Orgs.), Fear of crime and criminal victimization (pp. 131140). Stuttgart: Enke.
Wolfgang Bilsky, doutor em Psicologia pela Universidade Freiburg i.Br., é professor titular na Universidade de Münster.

Endereço para correspondência: Dr. Wolfgang Bilsky, Westfälische Wilhelms-Universität Münster, Fliednerstrasse 21, 48149 Münster, Deutschland. Tel.: 004925183 34198. Fax: 004925183 31343. E-mail: bilsky@psy.uni-muenster.de 